\title{
Pensamento computacional e processos cognitivos com pessoas idosas: revisão
}

\section{sistemática}

Computational thinking and cognitive processes with elderly people: systematic review

Pensamiento computacional y procesos cognitivos en personas mayores: revisión sistemática

Recebido: 30/08/2021 | Revisado: 05/09/2021 | Aceito: 08/09/2021 | Publicado: 11/09/2021

\author{
Emerson Rogério de Oliveira Junior \\ ORCID: https://orcid.org/0000-0001-5112-532X \\ Instituto Federal do Rio Grande do Sul, Brasil \\ E-mail: emerson.oliveira@sertao.ifrs.edu.br \\ Adriano Pasqualotti \\ ORCID: https://orcid.org/0000-0001-7544-9425 \\ Universidade de Passo Fundo, Brasil \\ E-mail: pasqualotti@upf.br
}

\begin{abstract}
Resumo
O pensamento computacional envolve processos cognitivos que buscam entender qual o problema pode ser resolvido e desenvolver possíveis soluções de forma eficiente e criativa. As pessoas idosas podem apresentar comprometimento cognitivo devido a fatores relacionados com a idade. $\mathrm{O}$ pensamento computacional é uma proposta adequada a ser aplicada nesta fase da vida. Neste estudo é apresentada uma revisão sistemática da literatura, cujo objetivo foi identificar as áreas de conhecimento em que o pensamento computacional, com foco nos processos cognitivos, está sendo aplicado às pessoas idosas. A busca foi realizada em maio de 2021, nas bases de dados eletrônicas disponibilizadas pela CAPES (acesso CAFe): Scopus, Web of Science, Scielo, Sbie, Eric, IEEE Xplore, Ovid, Science Direct, SpringerLink, SAGE Journals e Wiley Online Library. A pesquisa bibliográfica resultou em um total de 165 estudos e, após os critérios de exclusão, restaram 6 estudos para a síntese qualitativa. O método de pesquisa utilizado foi o PRISMA - Preferred Reporting Items for Systematic Reviews and Meta-Analyses. Os resultados apontam para a presença do pensamento computacional em diferentes áreas. Sua utilização apresentou aspectos bastante positivos quando tem seu foco voltado às pessoas idosas. O principal achado dos estudos analisados indica que a utilização do pensamento computacional por pessoas idosas possui um papel relevante para a estimulação cognitiva.
\end{abstract}

Palavras-chave: Habilidades de pensamento; Envelhecimento cognitivo; Resolução de problemas; Idosos.

\begin{abstract}
Computational thinking involves cognitive processes that seek to understand which problem can be solved and to develop possible solutions efficiently and creatively. Elderly people may have cognitive impairment due to agerelated factors. Computational thinking is a suitable proposal to be applied at this stage of life. In this study, a systematic literature review is presented, whose objective was to identify the areas of knowledge in which computational thinking, with a focus on cognitive processes, is being applied to the elderly. The search was carried out in May 2021, in electronic databases provided by CAPES (CAFe access): Scopus, Web of Science, Scielo, Sbie, Eric, IEEE Xplore, Ovid, Science Direct, SpringerLink, SAGE Journals and Wiley Online Library. The literature search resulted in a total of 165 studies, and after the exclusion criteria, 6 studies remained for the qualitative synthesis. The research method used was PRISMA - Preferred Reporting Items for Systematic Reviews and MetaAnalyses. The results point to the presence of computational thinking in different areas. Its use had very positive aspects when it has its focus on elderly people. The main finding of the analyzed studies indicates that the use of computational thinking by elderly people has a relevant role for cognitive stimulation.
\end{abstract}

Keywords: Thinking; Cognitive aging; Problem solving; Aged.

\section{Resumen}

El pensamiento computacional involucra procesos cognitivos que buscan comprender qué problema se puede resolver y desarrollar posibles soluciones de manera eficiente y creativa. Las personas mayores pueden tener deterioro cognitivo debido a factores relacionados con la edad. El pensamiento computacional es una propuesta adecuada para ser aplicada en esta etapa de la vida. Este estudio presenta una revisión sistemática de la literatura, cuyo objetivo fue identificar las áreas del conocimiento en las que se está aplicando el pensamiento computacional, con enfoque en los procesos cognitivos, a las personas mayores. La búsqueda se realizó en mayo de 2021, en bases de datos electrónicas puestas a disposición por CAPES (acceso a CAFe): Scopus, Web of Science, Scielo, Sbie, Eric, IEEE Xplore, Ovid, Science Direct, SpringerLink, SAGE Journals y Wiley Online Library. La búsqueda bibliográfica dio como resultado 
un total de 165 estudios y, tras los criterios de exclusión, quedaron 6 estudios para la síntesis cualitativa. El método de investigación utilizado fue PRISMA - Preferred Reporting Items for Systematic Reviews and Meta-Analyses. Los resultados apuntan a la presencia del pensamiento computacional en diferentes áreas. Su uso ha tenido aspectos muy positivos cuando tiene su foco en personas mayores. El principal hallazgo de los estudios analizados indica que el uso del pensamiento computacional por ancianos tiene un papel relevante para la estimulación cognitiva.

Palabras clave: Pensamiento; Envejecimiento cognitivo; Solución de problemas; Ancianos.

\section{Introdução}

Em 2060, um quarto da população $(25,5 \%)$ poderá ter mais de 65 anos. Nesse mesmo ano, o país terá 67,2 indivíduos com menos de 15 e acima dos 65 anos para cada grupo de 100 pessoas em idade da faixa de 15 a 64 anos (IBGE, 2018). De acordo com a Organização Mundial de Saúde, a crescente redução nas taxas de fertilidade e o aumento da longevidade irão assegurar o crescimento contínuo da população mundial e o consequente envelhecimento desta população (World Health Organization [WHO], 2015). O envelhecimento populacional traz consigo problemas de saúde que desafiam os sistemas de saúde e de previdência social. Envelhecer não significa necessariamente adoecer. A menos que exista doença associada, o envelhecimento está associado a um bom nível de saúde. Além disso, os avanços no campo da saúde e da tecnologia permitiram para a população com acesso a serviços públicos ou privados adequados, uma melhor qualidade de vida nessa fase. Com isso, é fundamental investir em ações de prevenção ao longo de todo o curso de vida (Miranda, Mendes \& Silva, 2016).

Considerando os problemas que acometem as pessoas com o avançar da idade, encontram-se na literatura trabalhos que tratam de: saúde (WHO, 2015; Piau, Rumeau \& Vellas, 2014; Rouillard et al. 2017); processo ensino/aprendizagem (Wing, 2006; Shute, Sun \& Asbell-Clarke, 2017) e socialização e bem-estar (Lee \& Park, 2020). O processo de envelhecimento deve ser pensado ao longo da vida e, assim, a prevenção se faz totalmente necessária. Agindo desta maneira, pode-se viver mais e com mais qualidade de vida.

Wing (2006, p.33) define pensamento computacional como "[...] um conjunto de habilidades e de atitudes universalmente aplicáveis em que qualquer pessoa, não apenas cientistas da computação estariam ansiosos para aprender a usar". Ainda de acordo com Wing (2006), PC não significa pensar como um computador, mas sim se envolver com processos cognitivos objetivando a resolução de problemas de forma eficiente e criativa. O PC inclui: buscar abordagens algoritmicas para domínios de problemas; estar preparado para se mover entre diferentes níveis de abstração e de representação; possuir familiaridade com decomposição; separação de interesses e modularidade. Isbell et al. (2009) defendem o PC com foco na prestação de serviços, interfaces e comportamentos que envolvem um papel mais central para a modelagem como um meio de formular relacionamentos e identificar fatores relevantes que podem vir a serem fontes de mudança. O PC nos permite analisar qualquer problema, por mais complexo que este se apresente, entendê-lo completamente e desenvolver soluções possíveis. Um problema complexo é aquele que, à primeira vista, não se sabe como resolver facilmente. Pode-se, então, apresentar as soluções de uma forma que um ser humano ou um computador (ou ambos) possam interpretar corretamente.

De acordo com Shute, Sun and Asbell-Clarke (2017, p.142), na última década, o PC se tornou um tópico muito importante na pesquisa e na prática educacional. Aparece uma grande quantidade de entradas em uma pesquisa geral no Google sobre sua definição, intervenções instrucionais e avaliação. Muitas dessas entradas sugerem que o PC se refere à codificação ou a aspectos específicos de programação, porém ele é muito mais do que apenas saber codificar um programa.

Existem quatro abordagens, também denominados de pilares, quando se está tratando do PC: decomposição, reconhecimento de padrões, abstração e algoritmos. A decomposição é caracterizada por dividir um sistema ou um problema complexo em partes menores e mais facilmente gerenciáveis. O reconhecimento de padrões requer a procura de semelhanças entre e dentro dos problemas. A abstração considera o foco apenas nas informações importantes, ignorando detalhes irrelevantes. O quarto pilar, que são os algoritmos, caracteriza-se pelo desenvolvimento de uma solução, passo a passo, para o 
problema ou a identificação das regras que devem ser empregadas para a resolução do problema em questão (Csizmadia et al., 2015, pp.6-8).

Assim, é possível analisar que, ao se tratar de um problema complexo, dividindo-o em partes menores e mais fáceis de atender, está sendo focada a fase de decomposição do PC. O reconhecimento de padrões ocorre quando são identificadas e aplicadas, nestas partes menores, soluções que foram anteriormente utilizadas para a resolução de outros problemas. A abstração ocorre quando são atendidas somente funções consideradas importantes, não sendo consideradas as demais particularidades deste problema. Complementando o processo, a fase de algoritmo é constituída quando forem definidos os procedimentos que serão executados, passo a passo, para a solução do problema em questão.

Investigando trabalhos que apresentam como foco a utilização de PC encontramos uma quantidade significativa de aplicações com foco principalmente na área da educação. Pode-se inferir que a maior parte das pesquisas relatadas tem como objetivo o ensino de algum tópico específico do conhecimento direcionado para crianças. Considerando o que foi exposto, o objetivo deste trabalho é identificar pesquisas que têm como foco a utilização do PC direcionada a idosos e identificar as áreas de concentração destes trabalhos. O mapeamento sistemático abordou a utilização do PC aplicado a pessoas idosas com o propósito de investigar em quais áreas do conhecimento as pesquisas com o PC vêm auxiliando as pessoas idosas, principalmente no que diz respeito a aspectos cognitivos, apresentando os resultados mais relevantes que forem encontrados. Para tanto, esta investigação tem a seguinte pergunta norteadora: Em quais áreas de aplicação as pessoas idosas utilizam o PC para a resolução de problemas?

\section{Metodologia}

A condução deste estudo de revisão sistemática segue as premissas indicadas no método PRISMA - Preferred Reporting Items for Systematic Reviews and Meta-Analyses (Moher, Liberati, Tetzlaff \& Altmann, 2009). Para Kitchenham \& Charters (2007), uma revisão sistemática da literatura é um meio de identificar, avaliar e interpretar as pesquisas disponíveis relevantes para uma determinada questão de pesquisa, área de assunto ou fenômeno de interesse.

\subsection{Estratégia de pesquisa}

Considerando a questão da pesquisa, alguns termos foram utilizados para a composição da string utilizada na busca. Torna-se importante indicar que, em maio de 2021, uma pesquisa no Google Scholar indicou 4.530.000 resultados para o termo de pesquisa elderly e 33.100 resultados para "Computational Thinking". Sinônimos foram consultados na literatura para compor a string de busca de acordo com a estratégia PICo (População, Intervenção, Contexto). Para o termo idoso também foram consultados sinônimos nos Descritores em Ciências da Saúde do DeCS (BIREME, 2021). Foram utilizadas palavraschave adicionais para expandir o escopo.

Para o escopo desta investigação foram utilizadas as bases de dados Scopus, Web of Science, Scielo e Sbie, com o objetivo de verificar o que se tem de pesquisas sobre a aplicação do PC envolvendo pessoas idosas. Assim, poderemos identificar as áreas de conhecimento que estão sendo atendidas por estes trabalhos. A Figura 1 representa a construção da string de busca resultante, sendo considerados os termos e a pergunta norteadora. 
Figura 1. Construção da string de busca.

Em quais áreas de aplicação as pessoas idosas utilizam o pensamento computacional para a resolução de problemas?

Pessoas idosas

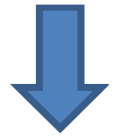

elderly

elderly person

oldest old

elder person

older adult(s)

elderly people
senior citizen(s)
elder(s) people
older old
older people

Pensamento computacional

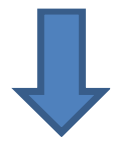

computational thinking

computational thinking method

computational thinking methodology

computational thinking technique

computational thinking and ageing

String de busca: (elderly OR "elderly people" OR "elderly person" OR "senior citizen" OR "senior citizens" OR "oldest old" OR "elder people" OR "elders people" OR "elder person" OR "older old" OR "older adult" OR "older adults" OR "older people") AND ("computational thinking" OR "computational thinking method" OR "computational thinking methodology" OR "computational thinking technique" OR "computational thinking and ageing"

Fonte: Os autores.

A busca foi realizada em maio de 2021, sem restrição de ano de publicação do trabalho, nas bases de dados eletrônicas disponibilizadas pela CAPES (acesso CAFe): Scopus, Web of Science, Scielo, Sbie, Eric, IEEE Xplore, Ovid, Science Direct, SpringerLink, SAGE Journals e Wiley Online Library. A expressão de pesquisa precisou ser adaptada em algumas bases de dados, considerando as especificidades de cada mecanismo de pesquisa, principalmente no que diz respeito à limitação no número de caracteres. Foram considerados artigos escritos em português, inglês e em espanhol. O filtro foi aplicado ao título, resumo, palavras-chave e texto completo.

\subsection{Critérios de elegibilidade}

Como critérios de elegibilidade para a execução desta RSL foram considerados: a) definição explícita da área de concentração e do problema a ser atendido; b) existência de resultados da aplicação do PC; c) público atendido composto ao todo ou em parte por pessoas idosas. Foram desconsiderados os artigos cujos dados estavam incompletos, bem como aqueles que, após esgotar todas as alternativas, não foram localizados os textos completos nas bases pesquisadas.

\subsection{Seleção dos estudos}

A seleção dos estudos foi estruturada em três etapas, a saber: a) Identificação: aplicação da string de pesquisa executada nas bases de dados selecionadas para a pesquisa; b) Triagem: títulos e resumos identificados por meio da estratégia de busca foram revisados com um exame preliminar para determinar se possuíam informações suficientes relacionadas ao objetivo desta revisão; c) Elegibilidade: estudos potencialmente relevantes, determinados como elegíveis com base no título ou resumo foram recuperados e avaliados com a leitura do texto completo, para estabelecer sua adequação aos critérios de elegibilidade. Dois investigadores realizaram todas as etapas da seleção dos estudos, simultaneamente e independentemente. Avaliações com resultados idênticos foram mantidas, avaliações divergentes passaram por uma nova análise conduzida em conjunto pelos dois investigadores, para chegar à decisão final. Quando os investigadores não conseguiram chegar a um acordo, a situação foi resolvida com o auxílio de um terceiro investigador. Estudos duplicados foram identificados e removidos. 


\subsection{Extração de dados}

Para a apresentação dos dados coletados foi utilizada uma tabela de extração padronizada, cujas linhas correspondem aos seguintes dados: a) Origem da investigação: apresentando o país originário da pesquisa; b) Caracterização da população atendida: considerando a quantidade de idosos participantes; c) Duração da investigação: quantidade de aplicações ou período de duração da pesquisa; d) Área de aplicação; e) Problema a ser solucionado; f) Métodos e as práticas empregadas; g) Coleta de dados.

\section{Resultados}

A pesquisa bibliográfica resultou em um total de 165 estudos, sendo 142 nas bases de dados e 25 em outras fontes e foram encontrados 2 estudos duplicados. Na sequência, 116 estudos foram excluídos durante a revisão de títulos e resumos, restando 49 artigos completos para a avaliação da elegibilidade. Destes, 43 estudos foram excluídos por quaisquer critérios de elegibilidade, resultando em 6 estudos incluídos para a síntese qualitativa. A Figura 2 representa o fluxo de seleção de estudos da pesquisa bibliográfica.

Figura 2. Fluxo de seleção de estudos da pesquisa bibliográfica (adaptação do diagrama PRISMA (Moher et al., 2009).

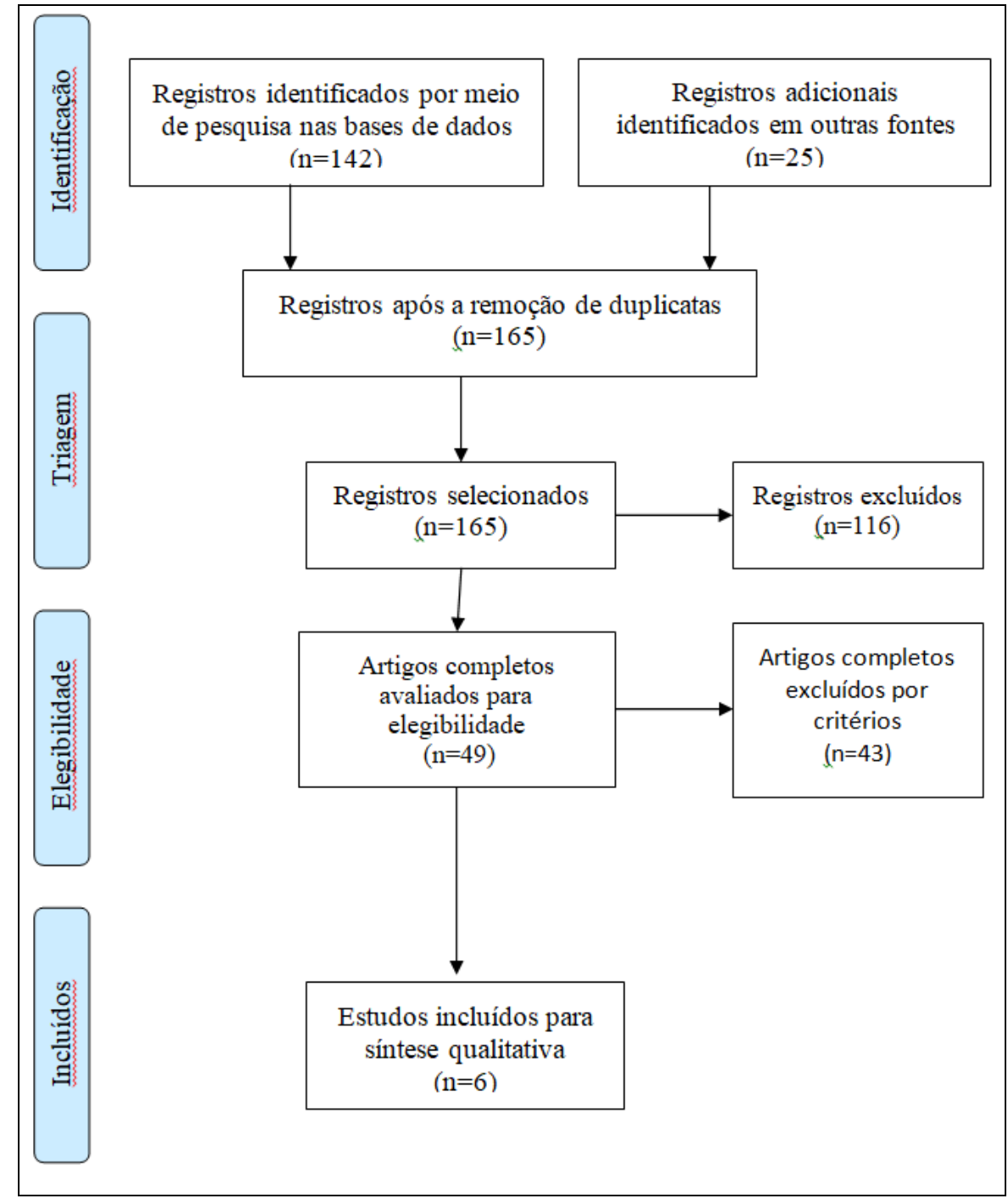

Fonte: Os autores. 
As principais características dos estudos relacionadas ao objetivo desta revisão sistemática da literatura estão sendo apresentadas e detalhadas no Quadro 1.

Quadro 1. Principais características dos estudos incluídos.

\begin{tabular}{|c|c|c|c|c|c|c|c|}
\hline & \multirow[b]{2}{*}{ Característica } & \multicolumn{6}{|c|}{ Estudos } \\
\hline & & $\begin{array}{l}\text { Lucena et } \\
\text { al., } 2020\end{array}$ & $\begin{array}{l}\text { Yang et } \\
\text { al., } 2011\end{array}$ & $\begin{array}{l}\text { Kale et al., } \\
2018\end{array}$ & $\begin{array}{c}\text { Lee e } \\
\text { Wong, } \\
2017\end{array}$ & $\begin{array}{l}\text { Azevedo e } \\
\text { Maltempi, } \\
2020\end{array}$ & $\begin{array}{c}\text { Lupşe, } \\
\text { Chirila e } \\
\text { Ciocârlie, } \\
2018\end{array}$ \\
\hline & Origem & Brasil & $\begin{array}{l}\text { Estados } \\
\text { Unidos }\end{array}$ & $\begin{array}{l}\text { Estados } \\
\text { Unidos }\end{array}$ & Malásia & Brasil & Romênia \\
\hline & erização da população & $\begin{array}{l}\text { idosos em } \\
\text { geral }\end{array}$ & $\begin{array}{l}\text { idosos em } \\
\text { geral }\end{array}$ & $\begin{array}{l}\text { professores } \\
\text { idosos }\end{array}$ & $\begin{array}{l}\text { idosos em } \\
\text { geral }\end{array}$ & $\begin{array}{c}\text { idosos } \\
\text { com } \\
\text { Parkinson }\end{array}$ & $\begin{array}{l}\text { idosos em } \\
\text { geral }\end{array}$ \\
\hline & Duração & $\begin{array}{c}24 \\
\text { aplicações }\end{array}$ & $\begin{array}{c}\text { dois } \\
\text { semestres }\end{array}$ & indefinido & indefinido & $\begin{array}{c}\text { dois } \\
\text { semestres }\end{array}$ & indefinido \\
\hline \multirow{5}{*}{ 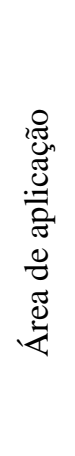 } & Computação & - & $\checkmark$ & - & - & $\checkmark$ & $\checkmark$ \\
\hline & Saúde & $\checkmark$ & - & - & $\checkmark$ & $\checkmark$ & - \\
\hline & Gerontologia & - & $\checkmark$ & - & - & - & - \\
\hline & Design Gráfico & - & $\checkmark$ & - & - & - & - \\
\hline & Ensino & - & - & $\checkmark$ & - & - & $\vee$ \\
\hline \multirow{6}{*}{ 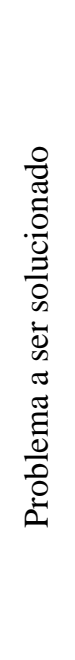 } & Estimulação cognitiva & $\checkmark$ & - & - & - & - & - \\
\hline & $\begin{array}{l}\text { Qualidade de vida ao } \\
\text { paciente com Parkinson }\end{array}$ & - & - & - & - & $\checkmark$ & - \\
\hline & $\begin{array}{l}\text { Aprendizagem de } \\
\text { tecnologias digitais }\end{array}$ & - & - & - & $\checkmark$ & - & - \\
\hline & $\begin{array}{l}\text { Criação de curso superior } \\
\text { de gerontecnologia }\end{array}$ & - & $\checkmark$ & - & - & - & - \\
\hline & Atividades pedagógicas & - & - & $\checkmark$ & - & - & - \\
\hline & Ensino de programação & - & - & - & - & - & $\checkmark$ \\
\hline$\sum_{i}^{\overline{0}} \tilde{0}$ & Atividades desplugadas* & $\checkmark$ & - & - & - & - & - \\
\hline
\end{tabular}




\begin{tabular}{|c|c|c|c|c|c|c|c|}
\hline & \multirow[b]{2}{*}{ Característica } & \multicolumn{6}{|c|}{ Estudos } \\
\hline & & $\begin{array}{c}\text { Lucena et } \\
\text { al., } 2020\end{array}$ & $\begin{array}{l}\text { Yang et } \\
\text { al., } 2011\end{array}$ & $\begin{array}{c}\text { Kale et al., } \\
2018\end{array}$ & $\begin{array}{c}\text { Lee e } \\
\text { Wong, } \\
2017\end{array}$ & $\begin{array}{c}\text { Azevedo e } \\
\text { Maltempi, } \\
2020\end{array}$ & $\begin{array}{c}\text { Lupşe, } \\
\text { Chirila e } \\
\text { Ciocârlie, } \\
2018\end{array}$ \\
\hline & Práticas pedagógicas & - & $\checkmark$ & - & - & - & - \\
\hline & Análise estatística & $\checkmark$ & - & $\checkmark$ & - & - & - \\
\hline & Interdisciplinaridade & - & $\checkmark$ & - & - & - & - \\
\hline & Projeto de algoritmos & - & - & - & - & - & $\checkmark$ \\
\hline & Gamificação & - & - & - & $\checkmark$ & - & - \\
\hline & Metodologias ativas & - & - & - & - & $\checkmark$ & - \\
\hline \multirow{6}{*}{ 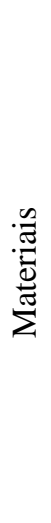 } & Jogos & - & - & - & $\checkmark$ & $\checkmark$ & - \\
\hline & Desenhos & $\checkmark$ & - & - & - & - & - \\
\hline & Exercícios & $\checkmark$ & - & - & - & - & - \\
\hline & Currículos & - & $\checkmark$ & - & - & - & - \\
\hline & Pesquisa & - & $\checkmark$ & $\checkmark$ & - & - & - \\
\hline & Manuais & - & - & - & - & - & $\checkmark$ \\
\hline \multirow{6}{*}{$\begin{array}{l}0 \\
\frac{0}{0} \\
\frac{\pi}{0} \\
\frac{0}{0} \\
\frac{\pi}{0} \\
\frac{\pi}{0}\end{array}$} & Observação & $\checkmark$ & - & - & - & $\checkmark$ & - \\
\hline & Questionário & $\checkmark$ & - & $\checkmark$ & $\checkmark$ & - & - \\
\hline & Entrevistas & - & - & - & - & $\checkmark$ & - \\
\hline & Imagens & - & - & - & - & $\checkmark$ & - \\
\hline & Transcrição de falas & - & - & - & - & $\checkmark$ & - \\
\hline & Painel de especialistas & - & $\checkmark$ & - & - & - & - \\
\hline
\end{tabular}

* Atividades desplugadas são atividades cujo objetivo é ensinar fundamentos (teóricos e práticos) da Ciência da Computação sem a utilização de computadores e afins.

Fonte: Os autores.

Percebe-se que a área de aplicação dos estudos selecionados é distinta, enfatizando que as possibilidades para a utilização do PC para o público idoso podem ser bastante variadas. Porém, podem ser destacadas duas áreas com maior concentração de trabalhos: Saúde (Lucena et al. (2020), Lee \& Wong (2017), Azevedo \& Maltempi (2020)) e Computação 
(Yang et al. (2011), Azevedo \& Maltempi (2020), Lupşe et al. (2018)). As demais áreas que apresentaram estudos foram Gerontologia (Yang et al. (2011)), Design Gráfico (Yang et al. (2011)) e Ensino (Kale et al. (2018)). Cabe aqui ressaltar que alguns trabalhos foram classificados em mais de uma área de aplicação devido ao fato de apresentar forte enfoque em mais de uma área. Quanto à duração dos estudos, também foi verificada grande disparidade em relação ao período e à quantidade de aplicações das atividades da pesquisa. Mesmo assim, metade dos trabalhos não apresentou estas informações devido ao fato de serem trabalhos considerados propostas em andamento.

No que se refere ao problema a ser solucionado com a utilização do PC para idosos houve bastante variação. O trabalho de Lucena et al. (2020) conecta o PC à estimulação cognitiva de pessoas idosas. Em sua pesquisa, Yang et al. (2011) propõem a criação de um curso superior, na Iowa State University, na área de Gerontecnologia, contendo conhecimentos das áreas de Ciência da Computação, Gerontologia e Design Gráfico. Kale et al. (2018) examinaram se a localização das escolas (rural versus urbana) e o nível de escolaridade (primário versus secundário) estão associados a variações nos níveis dos professores, cuja idade variava de 25 a 65 anos, quando considerada a utilização do PC em suas atividades docentes. Por sua vez, Lee and Wong (2017) apresentam um estudo sobre perspectivas computacionais para engajamento de idosos em Cidades Inteligentes utilizando o PC, relacionando-o com a experiência do usuário e com metodologias ágeis - teorias da Ciência da Computação. O trabalho de Azevedo e Maltempi (2020) tem como foco a utilização do PC na construção de jogos digitais e dispositivos de robótica destinados ao tratamento de limitações decorrentes da doença de Parkinson. Finalizando, Lupşe et al. (2018) propõem a construção de uma comunidade, conhecida como "Silver Code", composta por pessoas idosas com o objetivo de aprender programação e encorajar a interação com dispositivos eletrônicos, tendo o PC como uma das bases para aquisição deste conhecimento.

Em se tratando dos métodos apresentados nos trabalhos, foi verificado que Lucena et al. (2020) adaptaram ao público idoso, atividades desplugadas que foram criadas, inicialmente, para desenvolver o PC em crianças e realizou uma análise estatística para validar os seus achados. Yang et al. (2011) criaram o curso superior de gerontecnologia utilizando práticas pedagógicas específicas presentes em cursos de Ciência da Computação, Gerontologia e Design Gráfico, priorizando, também, a interdisciplinaridade necessária. Kale et al. (2018) fizeram uso de análise estatística para comprovar a diferença na utilização do PC nas atividades docentes dos professores investigados. No trabalho de Lee and Wong (2017) foi empregada a gamificação para a diversão e entretenimento dos idosos durante seu período de lazer pessoal e familiar, concetando-os com pessoas, a fim de torná-los mais felizes, se possível. Azevedo e Maltempi (2020) fizeram uso de metodologias ativas para a construção de dispositivos de robótica e de jogos eletrônicos. Finalizando, Lupşe et al. (2018) fizeram uso do projeto de algoritmos para permitir que os idosos pudessem interagir com dispositivos eletrônicos.

Os materiais utilizados nas investigações analisadas foram bem diferentes entre si. No trabalho de Lucena et al. (2020) foram utilizados exercícios contendo desenhos (atividades desplugadas) para estimular o PC nas pessoas idosas. Por sua vez, Yang et al. (2011) identificaram módulos necessários para a construção do novo curso de gerontecnologia através da análise dos currículos existentes dos cursos de Ciência da Computação, Gerontologia e Design Gráfico e aplicaram uma pesquisa para avaliar aspectos relacionados ao andamento do curso entre os alunos participantes. Na pesquisa de Kale et al. (2018) foi utilizada como material uma pesquisa para medir o acesso dos professores ao PC e às ferramentas de raciocínio computacional. Já, Lee and Wong (2017) fizeram uso de diferentes jogos para testar a hipótese de que as habilidades cognitivas e lógicas apresentam declínio mais lento do que a memória. Azevedo e Maltempi (2020) também fizeram uso de jogos, porém com foco na construção de jogos digitais e dispositivos de robótica destinados ao tratamento de Parkinson. Finalizando, Lupşe et al. (2018) propuseram manuais, como sendo guias estruturados de conhecimentos necessários para a comunidade Silver Code.

Quando são analisadas as diferentes maneiras dos procedimentos envolvidos na coleta dos dados apresentada nos trabalhos avaliados, verifica-se que o que mais aparece são questionário com três investigações (Lucena et al. (2020), Kale et 
al. (2018), Lee \& Wong (2017)) e observação com duas investigações (Lucena et al. (2020; Azevedo \& Maltempi (2020)). Com um trabalho cada, tem-se: entrevistas, imagens e transcrição de falas (Azevedo \& Maltempi (2020)) e painel de especialistas (Yang et al. (2011)). A pesquisa conduzida por Lupşe et al. (2018) não apresenta indicação de procedimentos envolvidos na coleta de dados por se tratar de uma proposta ainda em fase inicial, não tendo, portanto, resultados a serem apresentados e avaliados.

No trabalho de Lucena et al. (2020), questões desplugadas de PC adaptadas foram aplicadas em uma pesquisa de estimulação cognitiva constituída de dois grupos (controle e experimento) de idosos. Ambos os grupos foram submetidos a testes estatísticos com o objetivo de identificar mudança de desempenho presumindo, portanto, que a pontuação dos testes realizados após a intervenção será superior à dos testes realizados antes. Como parâmetro, foram comparadas as notas dos participantes antes e após o tratamento. Ao término das verificações nos grupos, ambos foram comparados quanto à mudança de desempenho. Como resultado, pode-se afirmar que, estatisticamente, não houve diferença entre as médias do pré-teste e pós-teste, isto é, não houve melhoria de desempenho significativo. Já no grupo de experimento, afirmou-se que, estatisticamente, houve diferença entre as médias do pré e pós-teste. Sendo assim, comprovou-se que houve uma melhoria de desempenho significativa apenas no grupo experimental, sendo um indício da eficácia da intervenção cognitiva baseada em atividades desplugadas de PC.

Analisando o trabalho de Yang et al. (2011), verifica-se que os resultados iniciais da primeira oferta do curso de Gerontecnologia são encorajadores em relação aos objetivos propostos, visto que foram observadas melhorias significativas na capacidade dos alunos para PC e habilidades analíticas em geral. Na primeira oferta, o curso teve 10 alunos inscritos. Foi verificada eficácia do curso na disseminação do PC. Os autores indicam que o curso produziu melhorias significativas dos alunos em capacidade de aplicar o PC para analisar e resolver problemas. Outro resultado interessante foi que alunos que não eram oriundos da Ciência da Computação apresentaram desempenho comparável aos desta área. Este fato demonstrou que alunos que não são da área de Ciência da Computação desenvolveram raciocínio lógico ao utilizarem PC. De acordo com os autores, um aspecto a ser melhorado foi o fato de que os conteúdos relacionados à gerontologia deveriam ter sido ensinados no decorrer de todo o semestre avaliado e não no ínicio do semestre. Esta situação trouxe à tona a situação de que os alunos não apresentaram melhora na compreensão das necessidades da população em envelhecimento (tema específico da área de gerontologia).

Considerando o estudo de Kale et al. (2018), os professores pesquisados acharam o PC relevante para atividades relacionadas ao ensino. Além disso, professores mais idosos consideraram o PC relevante não apenas para o ensino, mas também para suas vidas pessoais. Em relação às tecnologias em sala de aula, os professores indicaram que eles "podem solicitar a instalação do software", indicando que a maioria dos professores desconhecia o termo "pensamento computacional" - confundindo-o com um software. Uma constataçao importante desta pesquisa foi que, quanto maior a quantidade de tempo (em anos) de ensino, os professores menos habilidosos pouco usavam ferramentas de PC e a motivação para o seu uso não variou com base na localizaçao das escolas (urbana ou rural). Houve um efeito de interação estatisticamente significativo entre ruralidade e nível de escolaridade sobre as diferentes tecnologias a que os professores tiveram acesso na escola. Também houve um efeito de interação estatisticamente significativo entre ruralidade e nível em habilidades no emprego do PC. Finalizando, professores do ensino médio usaram ferramentas de PC com menos frequência do que professores do ensino fundamental.

O trabalho de Azevedo e Maltempi (2020) tem como princípio a utilização do PC na construção dos jogos e dispositivos de robótica que são utilizados no tratamento de idosos acometidos com Parkinson. Como resultados, foi verificado que atenderam ao objetivo, uma vez que, segundo depoimentos de pessoas idosas atendidas pelo projeto, indicaram que: "ajudam a mexer e a usar a mente" e "as atividades aqui são uma interação importante, porque auxilia nos movimentos, [...] 
ajuda a despertar a alegria através da interação com os alunos". Através destes depoimentos, é verificado que a utilização do PC sugere auxílio nos problemas que acometem pessoas que apresentam a doença de Parkinson.

Tanto os trabalhos desenvolvidos por Lee and Wong (2017), que apresentam um estudo sobre perspectivas computacionais para engajamento de idosos em cidades inteligentes, quanto a investigação de Lupşe et al. (2018), que propõe a construção da comunidade Silver Code, composta por pessoas idosas com o objetivo de aprender programação e encorajar a interação com dispositivos eletrônicos, são trabalhos preliminares e, por esta razão, não apresentaram nenhum resultado. Um resumo dos principais resultados apresentados pelos estudos investigados que dizem respeito às pessoas idosas está sendo apresentado no Quadro 2.

Quadro 2. Principais resultados dos estudos incluídos para pessoas idosas.

\begin{tabular}{|c|c|}
\hline Estudo & Resultados \\
\hline Lucena et al. (2020) & Foi verificada eficácia da intervenção cognitiva baseada em atividades desplugadas de PC. \\
\hline Yang et al. (2011) & $\begin{array}{l}\text { Houve melhoria significativa na capacidade dos alunos para PC e habilidades analíticas em geral; } \\
\text { melhorias significativas dos alunos em capacidade de aplicar o PC para analisar e resolver problema; } \\
\text { alunos não eram oriundos da Ciência da Computação apresentaram desempenho comparável aos desta } \\
\text { área; conteúdos relacionados à gerontologia deveriam ter sido ensinados no decorrer de todo o } \\
\text { semestre avaliado e não no início do semestre. }\end{array}$ \\
\hline Kale et al. (2018) & $\begin{array}{l}\text { Os professores acharam o PC relevante para suas vidas e ensino; professores mais idosos consideraram } \\
\text { o PC relevante para o ensino e, também, para suas vidas; professores indicaram que eles "podem } \\
\text { solicitar a instalação do software"; a maioria dos professores desconhecia o termo "pensamento } \\
\text { computacional"; quanto maior o número de anos de ensino, os professores menos habilidosos pouco } \\
\text { usavam ferramentas de PC e a motivação para o seu uso não variou com base na localização das } \\
\text { escolas (urbana ou rural); houve relação entre ruralidade e nível de escolaridade sobre as diferentes } \\
\text { tecnologias a que os professores tiveram acesso na escola; houve relação entre ruralidade e nível em } \\
\text { habilidades no emprego do PC; professores do ensino médio usaram ferramentas de PC com menos } \\
\text { frequência do que professores do ensino fundamental. }\end{array}$ \\
\hline Lee and Wong (2017) & Por se tratar de trabalho preliminar, não apresenta nenhum resultado. \\
\hline Azevedo e Maltempi (2020) & $\begin{array}{l}\text { Foi indicado que a utilização do PC sugere auxílio nos problemas que acometem pessoas que } \\
\text { apresentam Parkinson. }\end{array}$ \\
\hline Lupşe et al. (2018) & Por se tratar de trabalho preliminar, não apresenta nenhum resultado. \\
\hline
\end{tabular}

Fonte: Os autores.

\section{Discussão}

Retomando, esta investigação foi realizada considerando a seguinte pergunta norteadora: Em quais áreas de aplicação as pessoas idosas utilizam o PC para a resolução de problemas? Com um mapeamento das diferentes aplicações do PC em atividades relacionadas às pessoas idosas, é útil analisar as características de cada aplicação, bem como seus resultados. Essa revisão sistemática encontrou indicadores de que a utilização do PC pode (e deve) ser aplicado a atividades relacionadas com idosos por ser mais uma forma de estimulação cognitiva (Lucena et al. (2020), Azevedo \& Maltempi (2020), Lupşe et al. (2018)); por ser uma estratégia eficaz para modelar soluções e resolver problemas de maneira eficiente (Lucena et al. (2020), 
Yang et al. (2011), Kale et al. (2018), Azevedo \& Maltempi (2020)); por ser uma habilidade essencial para a sociedade moderna (Yang et al. (2011), Kale et al. (2018), Lee \& Wong (2017)); por contribuir com seu engajamento e integração social (Yang et al. (2011), Kale et al. (2018), Lee \& Wong (2017), Lupşe et al. (2018)) e por melhorar a autoconfiança (Lucena et al. (2020), Kale et al. (2018), Lee \& Wong (2017), Azevedo \& Maltempi (2020), Lupşe et al. (2018)).

Quando avaliamos a presença dos pilares do processo do PC, conforme indicado por Wing (2006), decomposição, reconhecimento de padrões, abstração e algoritmos, nos artigos avaliados nesta RSL, verifica-se que Lucena et al. (2020) indicam que fizeram uso de atividades desplugadas existentes baseadas em PC, porém sem apresentar nenhum detalhamento quanto à caracterização destas atividades nos pilares. Yang et al. (2011) apontam a presença dos pilares do PC em quase todas as etapas da formulação do curso superior de Gerontecnologia, tendo sido indicado o PC como uma das habilidades fundamentais dos futuros alunos deste curso. Kale et al. (2018) explicitam que o PC se relaciona às habilidades dos professores avaliados no trabalho para usar a tecnologia na vida cotidiana, porém não indicam especificamente como avaliam a caracterização das habilidades do PC, em cada um dos seus pilares. Lee and Wong (2017) indicam que, através do emprego do PC, a computação estende a codificação para aplicação a problemas reais e é neste contexto que os autores propõem a criação da cidade inteligente, porém não apresentam a maneira com que atende aos pilares do PC. Azevedo e Maltempi (2020) apontam que o emprego dos pilares do PC aparece em atividades como: a investigação da estrutura de programação e ideias matemáticas envolvidas, a argumentação acerca do funcionamento desta estrutura, a simulação de diferentes resultados, a formulação de hipótese de descoberta e a descoberta de valores estratégicos. Lupşe et al. (2018) apresentam, em seu trabalho, aspectos dos pilares do PC. Esta informação pode ser localizada na estrutura do guia de programação, proposto para ser utilizado pela comunidade Silver Code. Uma das unidades deste guia indica com riqueza de detalhes como se pode obter a decomposição, o reconhecimento de padrões, a abstração e o design de algoritmos.

Cabe salientar que os estudos envolvidos nesta revisão sistemática confirmaram vários tópicos apontados na literatura sobre o PC com foco em pessoas idosas, conforme descrito na revisão da literatura apresentada. Essa relação é evidenciada, principalmente no que diz respeito à utilização do PC para atender às especificidades de pessoas idosas em diferentes áreas de aplicação, mostrando que o PC tem muito a contribuir para melhorar a qualidade de vida destas pessoas.

\section{Considerações Finais}

No que tange às limitações do estudo, cabe ressaltar que, para a sua realização, foram utilizados termos de busca específicos, que podem não ter sido capazes de capturar a totalidade dos artigos sobre o assunto proposto. Os estudos revisados apresentam contextos, itens e tamanhos diferentes entre si, o que dificulta a realização de um mapeamento padronizado. Uma dificuldade enfrentada para a realização da seleção dos estudos para esta revisão sistemática da literatura foi a escassez de trabalhos que consideram o emprego do PC para aplicações envolvendo pessoas idosas. Por esta razão, há indicações de que se trata de uma área do conhecimento que ainda tem muito para ser explorada. Porém, mesmo com a falta de trabalhos científicos mais específicos, foi possível identificar e explorar contribuições de trabalhos envolvendo algumas áreas de aplicação do PC. Considerando os achados científicos apresentados nos trabalhos analisados e detalhados nesta revisão sistemática da literatura, comprovou-se que a utilização do PC por pessoas idosas possui um papel relevante para ser aplicado com foco na estimulação cognitiva.

\section{Referências}

Angeli, C., \& Giannakos M. N. (2020). Computational thinking education: Issues and challenges. Computers in Human Behavior, 105:106185 https://doi.org/10.1016/j.chb.2019.106185. 
Azevedo, G. T. \& Maltempi, M. (2020). Processo de Aprendizagem de Matemática à luz das Metodologias Ativas e do Pensamento Computacional. Ciência e Educação, 26: e20061.

Bireme. DeCS/MeSH. (2017). Descritores em Ciências da Saúde. https://decs.bvsalud.org/.

Csizmadia, A., Curzon, P., Dorling, M., Humphreys, S., Ng, T., Selby, C., \& Woollard, J. (2015). Computational thinking - a guide for teachers.

IBGE - Instituto Brasileiro de Geografia e Estatística. (2018). Projeção da População 2018: número de habitantes do país deve parar de crescer em 2047. https://agenciadenoticias.ibge.gov.br/agencia-sala-de-imprensa/2013-agencia-de-noticias/releases/21837-projecao-da-populacao-2018-numero-de-habitantesdo-pais-deve-parar-de-crescer-em-2047

Isbell, C. L., Stein, L. A., Cutler, R., Forbes, J., Fraser, L., Impagliazzo, J., Proulx, V., Russ, S., Thomas, R. \& Xu, Y. (2009). (re)defining computing curricula by (re)defining computing. ACM SIGCSE Bulletin, 41(4), 195-207.

Kale, U., Akcaoglu, M., Cullen, T. \& Goh, D. (2018). Contextual Factors Influencing Access to Teaching Computational Thinking. Computers in the Schools, $35(2), 69-87$.

Kitchenham, B. \& Charters, S. (2007). Guidelines for performing Systematic Literature Reviews in Software Engineering. Information and Software Technology, 51(1), 7-15.

Lee, C. \& Wong, K. D. (2017). Developing community-based engagement in Smart Cities: A design-computational thinking approach. Proc. IEEE International Conference on Industrial Engineering and Engineering Management (IEEM), 832-836.

Lee, E. \& Park, S. (2020). Immersive Experience Model of the Elderly Welfare Centers Supporting Successful Aging. Frontiers in Psychology, 11: 8.

Lucena, D. A., Nunes, I. D., Rodrigues, R. S. \& Souza, D. R. O. (2020). Adaptações em atividades de Pensamento Computacional para estimulação cognitiva em idosos. Anais XXXI Simpósio Brasileiro de Informática na Educação (SBIE 2020), 1533-1542.

Lupşe, O., Chirila, C. \& Ciocârlie, H. (2011). Programming Concepts in the Silver Code Guide for Elders. Proc. IEEE 12 ${ }^{\text {th }}$ International Symposium on Applied Computational Intelligence and Informatics (SACI), 137-142.

Miranda, G. M. D., Mendes, A. C. G. \& Silva, A. L. A. (2016). O envelhecimento populacional brasileiro: desafios e consequências sociais atuais e futuras. Revista Brasileira de Geriatria e Gerontologia, 19(3), 507-519.

Moher, D., Liberati, A., Tetzlaff, J. \& Altmann, D. G. (2009). Preferred Reporting Items for Systematic Reviews and Meta-Analyses: The PRISMA Statement. PLoS Medicine, 6(7): e1000097.

Piau, A., Campo, E., Rumeau, P. \& Vellas, B. (2014). Aging society and gerontechnology: a solution for an independent living?. The Journal of Nutrition, Health \& Aging, 18(1), 97-112.

Rouillard, M., Audiffren, M., Albinet, C., Bahri, M. A., Garraux, G. \& Collette, F. (2017). Contribution of four lifelong factors of cognitive reserve on late cognition in normal aging and Parkinson's disease. Journal of Clinical and Experimental Neuropsychology. 39(2), $142-162$.

Shute, V. J.; Sun, C. \& Asbell-Clarke, J. (2017). Demystifying Computational Thinking. Educational Research Review, 22, 142-158.

Yang, H., Martin, P., Satterfield, D., Babbitt, R., Wong, J., Shelley, M. \& Chang, C. (2011). A Novel Interdisciplinary Course in Gerontechnology for Disseminating Computational Thinking. Proc. $41^{\text {th }}$ ASEE/IEEE Frontiers in Education Conference Political Science Presentations and Posters.

Wing, J. M. (2006). Computational thinking. Communications of the ACM, 49(3), 33-35.

World Health Organization. (2015). World report on ageing and health. World Health Organization. https://apps.who.int/iris/handle/10665/186463. 